\title{
Effect of fault damage zones on long-term earthquake behavior on mature strike-slip faults
}

${ }^{1}$ University of Michigan, Department of Earth and Environmental Sciences ${ }^{2}$ GNS Science, New Zealand

\section{Key Points:}

- Fully dynamic earthquake cycle simulations show persistent heterogeneous stress distribution generated by low-velocity fault zone waves

- Faults surrounded by low-velocity damage zones lead to more complex patterns of earthquakes

- Shallower damage zones tend to have shallower earthquake hypocenters

This paper is a non-peer reviewed preprint submitted to JGR:Solid Earth.

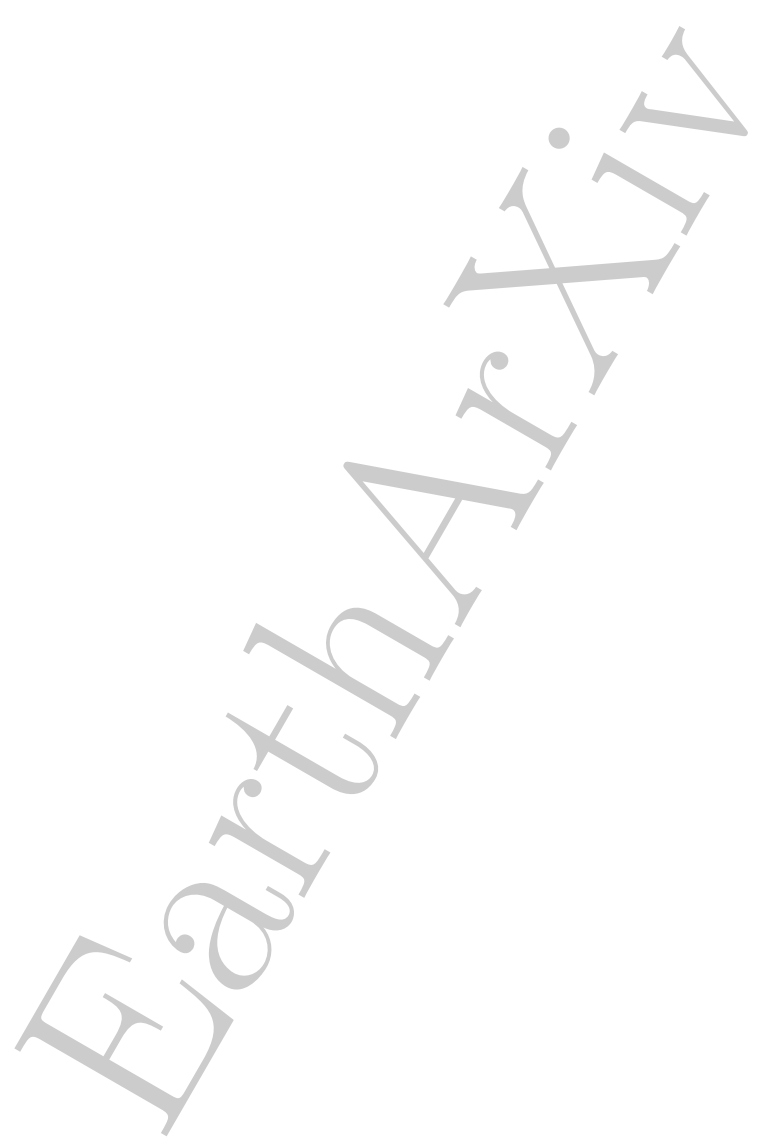

Corresponding author: Prithvi Thakur, prith@umich.edu 


\begin{abstract}
Mature strike-slip faults are usually surrounded by a narrow zone of damaged rocks characterized by low seismic wave velocities. Observations of earthquakes along such faults indicate that seismicity is highly concentrated within this fault damage zone. However, the long-term influence of the fault damage zone on complete earthquake cycles, i.e., years to centuries, is not well understood. We simulate aseismic slip and dynamic earthquake rupture on a vertical strike-slip fault surrounded by a fault damage zone for a thousandyear timescale using observations along major strike-slip faults, e.g., San Andreas Fault, as constraints on fault zone material properties and geometry. We find that dynamic wave reflections, whose characteristics are strongly dependent on the width and the rigidity contrast of the fault damage zone, have a prominent effect on the stressing history of the fault. The presence of elastic damage can explain, in part, the variability in the earthquake sizes and hypocenter locations along a single fault, which vary with fault damage zone depth, width and rigidity contrast from the host rock. The depth extent of the fault damage zone has a pronounced effect on the earthquake hypocenter locations, and shallower fault damage zones favor shallower hypocenters with a possible bimodal distribution of seismicity along depth. Our findings also suggest significant fault damage zone effects on the hypocenter distribution when the fault damage zone penetrates to the nucleation sites of earthquakes. Therefore the depth distribution of seismicity in mature strike-slip faults is likely influenced by both lithological (material) and rheological (frictional) boundaries.
\end{abstract}

\title{
Plain Language Summary
}

Large strike-slip earthquakes tend to create a zone of fractured network surrounding the main fault. This zone, referred to as a fault damage zone, becomes highly localized as the fault matures, spanning several hundred meters in width. The influence of this fault damage zone on earthquake characteristics remains elusive since we do not have enough long-term observations along a single fault. We use numerical simulations to examine the behavior of earthquake nucleation and rupture dynamics on a fault surrounded by a damage zone over a thousand-year timescale. Our simulations reveal that the reflection of seismic waves from the fault damage zone boundaries leads to complexity in earthquake sequences, such as variability in earthquake locations and sizes. We also show that a shallow fault damage zone produces shallower earthquakes with the earthquake depths centered around two locations (bimodal), as opposed to a deep fault damage zone with the earthquake depths centered around a single location (unimodal). Our study suggests that imaging the geometry and physical properties of fault damage zones could potentially give us clues about depths of future earthquakes and improve earthquake probabilistic hazard assessment.

\section{Introduction}

Natural faults are often approximated as a single plane of intense deformation, macroscopically seen as a principal slip surface. However, geological (e.g., F. Chester and Logan (1986); F. M. Chester et al. (1993); Lockner et al. (2011)), geophysical (e.g., Li and Leary (1990); Unsworth et al. (1997); Lewis and Ben-Zion (2010)), and geodetic (e.g., Fialko et al. (2002)) observations delineate faults as a geometrically complex network of multiple slip surfaces and fractures, with a nested hierarchy of increasing deformation towards the principal slip surface (Fig. 1). These damaged rocks exhibit a dense network of fractures which can be macroscopically approximated as an elastic zone with reduced shear modulus and seismic velocities (F. M. Chester et al., 1993; Harris \& Day, 1997). The damage zones, exhibiting sharp contrast of seismic velocities with respect to the host rocck, are capable of trapping seismic waves. The fault damage zone can potentially promote complex stress distribution along faults due to its pronounced dynamic effect on earthquake rupture nucleation and propagation (e.g., Harris and Day (1997); Huang and Ampuero (2011); Huang et al. (2014); Ma and Elbanna (2015); Albertini and Kammer (2017); Weng et al. (2016); Huang (2018)). 
(b) Fault Zone Schematic

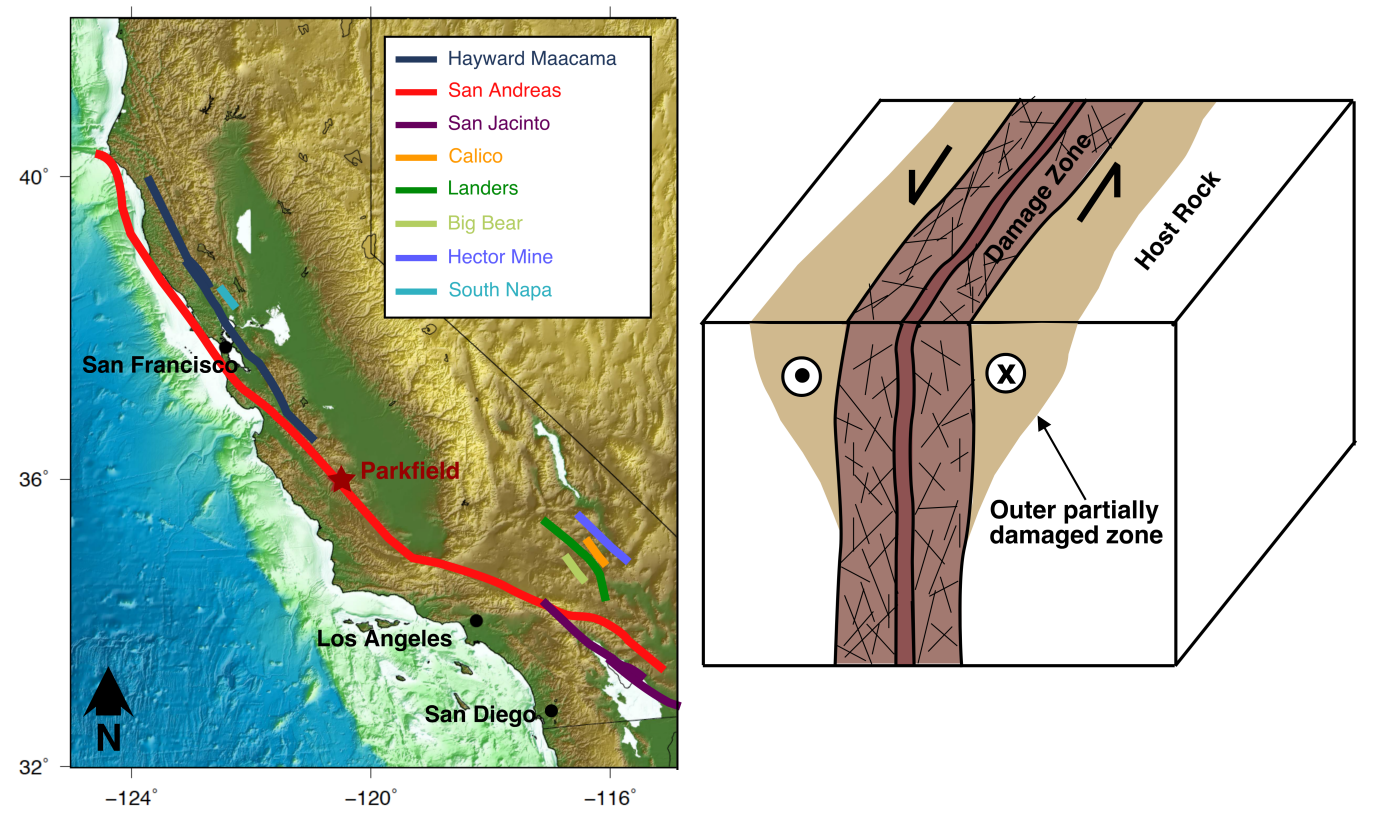

(a) Fault Damage Zones in California

We aim to understand the dynamic effects of the fault damage zone over earthquake cycles, which include interseismic slip, earthquake nucleation, rupture propagation, and postseismic slip, and study its influence on the variability in earthquake sizes, recurrence intervals and stressing history of the fault.

N 
we consider fully dynamic continuum models with fault damage zone surrounding mature strike-slip faults. Using fully dynamic earthquake cycle simulations, Kaneko et al. (2011) showed that a fault-parallel, narrow damage zone causes a reduction in the nucleation size of the earthquakes and amplification of slip rates during dynamic earthquake events. Despite multitude of studies documenting the effects of fault damage zones on single rupture (Harris \& Day, 1997; Huang \& Ampuero, 2011; Huang et al., 2014), thêir long-term effects on earthquake sequences are not well understood, partially owing to a lack of seismological records over centuries.

We model earthquake sequences with full inertial effects on a two-dimensional vertical strike-slip fault surrounded by a fault damage zone. The constitutive response of the fault is governed by laboratory derived rate-and-state friction laws (Dieterich, 1979; Ruina, 1983). This fully dynamic modeling approach can simulate interseismic slip, earthquake nucleation, rupture propagation and postseismic deformation during multiple seismic cycles in a single computational framework (e.g.,Lapusta et al. (2000); Kaneko et al. (2011); Barbot et al. (2012); Jiang and Lapusta (2016)). We investigate how the wave reflections from fault damage zone influences the long-term stress evolution and explain the variability in earthquake magnitudes and hypocenter locations. We show that the variability in earthquake hypocenter is significant only in the cases where the damage zone truncates close to the nucleation site or extends beyond the nucleation zone, suggesting that frictional and rheological effects may be a dominant mechanism for hypocenter variability when the damaged structure is very shallow. Our results also provide a possible explanation for the bimodal depth distribution of seismicity observed along mature strike-slip faults with shallow fault damage zone structures. We describe the observed geometry and material properties of the fault damage zone along the San Andreas Fault that inspire the design of our simulations in section 2. The two-dimensional model setup, model assumptions, friction laws, and simulation methodology are presented in section 3. We demonstrate the effects of the fault damage zone with varying widths and rigidity contrasts on the variability of earthquake magnitudes and hypocenters in section 4 .

\section{Observed dimension and material properties of fault damage zones}

Fault damage zones can be delineated using potential field methods and seismic observations based on trapped waves within the damaged zone. Seismic reflections, magnetotelluric and resistivity surveys along the Parkfield segment of San Andreas Fault reveal a $500 \mathrm{~m}$ wide and $4 \mathrm{~km}$ deep fault damage zone (Unsworth et al., 1997). This study also suggests a presence of a deeper fault zone whose property is not well resolved, and a shallow $5 \mathrm{~km}$ wider damage zone surrounding the $\sim 500 \mathrm{~m}$ wide damage zone, representing a flower structure. Other studies along San Jacinto Fault Zone and San Andreas Fault Zone (e.g., Li and Vernon (2001); Wu et al. (2010)) also indicate that the low-velocity zone may extend to seismogenic depths. Fault zone trapped wave studies along the Parkfield segment (Lewis \& Ben-Zion, 2010) indicate a $3 \mathrm{~km}$ to $5 \mathrm{~km}$ deep, $150 \mathrm{~m}$ to $300 \mathrm{~m}$ wide fault damage zone, with a potentially nested fault zone extending up to $7 \mathrm{~km}$ to $10 \mathrm{~km}$. Geologic interpretations on the same region from the SAFOD cores (Lockner et al., 2011) delineate a $\sim 200 \mathrm{~m}$ wide fault damage zone at $2.7 \mathrm{~km}$ depth. A detailed 3-D seismic wave velocity map (Thurber et al., 2003) also reveals a several hundred meters wide fault zone structure at about $5 \mathrm{~km}$ to $8 \mathrm{~km}$ depth. The shear wave velocity contrast between the host rock and the fault damage zone is found to be around $10 \%$ to $60 \%$ (table 1 in Huang et al. (2014) and references therein). Most of these studies report variations in fault damage zone structure along fault strike. We summarize the observed fault damage zone geometry along the Parkfield segment in Table 1. Based on this review, it is clear that the fault damage zone width spans several hundred meters, whereas the depth extent is more debatable since the narrow damage zone is more difficult to resolve at depth. We use these observations to guide our model setup as described in the following section. 
Table 1. Geometry of fault damage zone along Parkfield segment of San Andreas Fault as constrained by different studies.

\begin{tabular}{|c|c|c|c|}
\hline References & Geometry & Width Inference & Depth Inference \\
\hline $\begin{array}{l}\text { Resistivity and MT } \\
\text { (Unsworth et al., 1997) }\end{array}$ & $\begin{array}{l}\text { Wide at the top, } \\
\text { narrow at depth }\end{array}$ & $\begin{array}{r}500 \mathrm{~m} \text { for inner damage } \\
5 \mathrm{~km} \text { for outer damge }\end{array}$ & $\begin{array}{c}4 \mathrm{~km}, \text { with a deeper } \\
\text { damage zone less resolved }\end{array}$ \\
\hline $\begin{array}{l}\text { Trapped seismic waves } \\
\text { (Lewis \& Ben-Zion, 2010) }\end{array}$ & $\begin{array}{l}\text { Tabular low velocity } \\
\text { zone }\end{array}$ & $150 \mathrm{~m}$ to $300 \mathrm{~m}$ & $5 \mathrm{~km}$ to $7 \mathrm{~km}$ \\
\hline $\begin{array}{l}\text { Seismic wave velocities } \\
\text { (Thurber et al., 2003) }\end{array}$ & $\begin{array}{c}\text { Wide at the top } \\
\text { and at seismogenic depth, } \\
\text { narrow in between }\end{array}$ & $500 \mathrm{~m}$ to & $8 \mathrm{~km}$ \\
\hline $\begin{array}{l}\text { Geology: SAFOD } \\
\text { (Lockner et al., 2011) }\end{array}$ & Tabular & & $2 \mathrm{~km}$ \\
\hline
\end{tabular}

\section{Methodology}

\subsection{Model Description}

We consider a two-dimensional strike-slip fault embedded in an elastic medium with mode III rupture Fig. 2. This implies that the fault motion is in and out of the plane and only the depth variations of parameters are considered. The top boundary is stress-free and represents earth's free surface. The other three boundaries are absorbing boundaries that allow the waves to pass through. Since our model is symmetric across fault, we restrict the computational domain to only one side of the fault. Our domain extends to $48 \mathrm{~km}$ depth, where the top $24 \mathrm{~km}$ of the fault is bordered at the bottom by a region constantly slipping at $35 \mathrm{~mm} \mathrm{yr}^{-1}$. This represents the tectonic plate motion that loads the fault and accumulates stresses. The seismogenic zone extends from $2 \mathrm{~km}$ to $15 \mathrm{~km}$, which is locked during the interseismic period and capable of hosting earthquakes. The rest of the fault creeps aseismically. Earthquakes are captured in our simulations when the maximum slip velocity on the fault exceeds the threshold of $0.001 \mathrm{~mm} \mathrm{~s}^{-1}$. This model is inspired by the San Andreas fault, and is similar in setup to Lapusta et al. (2000) and Kaneko et al. (2011).

The fault damage zone is modeled as an elastic layer with lower seismic wave velocities compared to the host rock. We will focus on how the geometry, spatial extent, and damage intensity of this fault damage zone influence the earthquake sequence behavior. We consider four different scenarios: (I) a homogeneous elastic medium as a reference model, (II, III) a medium with a sharp, narrow fault damage zone with various depths, widths and velocity contrasts that extends throughout the seismogenic depth in model II and truncates at a shallow depth in model III, and (IV) a flower structure in which a narrow fault damage zone extending through the domain surrounded by a wider, trapezium-shaped fault damage zone truncated at a shallow depth (Fig. 2). In natural settings, the outer trapeziumshaped fault damage zone may not have a sharp boundary at depth but may show a smooth transition because its structure is more diffused than the inner fault damage zone. We use a sharp boundary as an approximation of the flower structure in order to highlight the effects of dynamic wave reflections. These four sets of models are described in Fig. 2. We vary the width $(H)$ and shear wave velocity $\left(c_{s}\right)$ contrast of the fault damage zone in the Model (II) and the depth $(D)$ in model III to study their effects on earthquake sizes and hypocenters (Fig. 2a). The choices of ' $H$ ' and ' $c_{s}$ ' are shown in Fig. 3. We choose four different values of ' $D$ ' including two depths $(6 \mathrm{~km}$ and $8 \mathrm{~km})$ shallower than the nucleation 

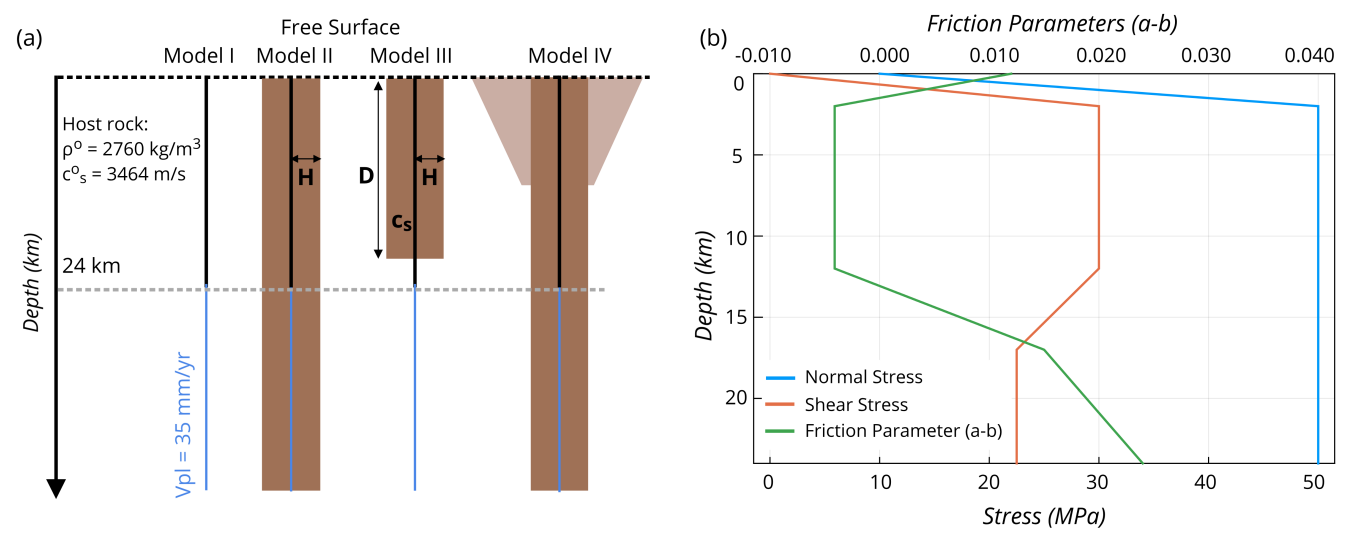

Figure 2. (a) Model description of four different scenarios. We consider a vertical strike-slip fault $24 \mathrm{~km}$ deep loaded from below by a plate motion rate of $35 \mathrm{~mm} \mathrm{yr}^{-1}$. Model I: Homogeneous medium used as a reference model. Model II: A narrow fault damage zone extending throughout the seismogenic zone. Model III: A narrow fault damage zone truncating at a shallower depth, and Model IV: Two-dimensional approximation of flower structure damage. (b) Friction parameters $(a-b)$ and initial stresses along the fault dip. The seismogenic zone, i.e., the velocity weakening region, is the overstressed patch between 2 and $15 \mathrm{~km}$ depth.

site in the homogeneous medium, one depth intersecting the nucleation zone $(10 \mathrm{~km})$ and one depth extending beyond the nucleation zone $(12 \mathrm{~km})$. In the Model (IV), the outer, wider fault damage zone has a shear wave velocity reduction of $20 \%$ compared to the host rock, while the inner one has a $40 \%$ reduction. The second and third models are inspired by the geological and geophysical observations of the San Andreas fault zone as discussed in section 2, and the fourth model is inspired by the classic flower structure of fault damage zones (Sibson, 1977; Unsworth et al., 1997; Caine et al., 1996; Pelties et al., 2015; Perrin et al., 2016).

\subsection{Friction Laws}

We use laboratory-derived rate and state friction laws to describe the fault slip (Dieterich, 1979; Ruina, 1983; C. H. Scholz, 1998). This friction law relates the shear strength $(T)$ on the fault to the slip $\operatorname{rate}(\dot{\delta})$ as follows:

$$
\mathcal{T} T=\bar{\sigma}\left[f_{o}+a \ln \left(\frac{\dot{\delta}}{\dot{\delta_{o}}}\right)+b \ln \left(\frac{\dot{\delta_{o}} \theta}{L}\right)\right]
$$

where $\bar{\sigma}$ is the effective normal stress (the difference between lithostatic stress and the pore fluid pressure), $f_{o}$ is a reference friction coefficient corresponding to a reference slip rate $\dot{\delta}_{o}$, and $a \& b$ are empirical constants dependent on the mechanical and thermal properties of the contact surface. The parameter $\theta$ is a state variable interpreted as the average lifetime of the surface in contact, and $L$ is the characteristic distance over which the contact surface slips. The term $a \ln \left(\frac{\dot{\delta}}{\dot{\delta}_{o}}\right)$ is called the direct effect term, and the term $b \ln \left(\dot{\delta_{o}} \theta / L\right)$ is the state evolution term (Eq. 1). The state variable $\theta$ is intuitively hard to understand, so the term $f_{o}+b \ln (\dot{\delta} \theta / L)$, referred to as $\psi$ is commonly used to interpret the state evolution term and is defined as the current absolute offset of the friction coefficient. The frictional stability of faults is determined by two frictional parameters, $L$ and $(a-b)$. Depending on the value of $(a-b)$, we can have an unstable slip for a steady state velocity weakening frictional regime $(a-b)<0$, or a stable sliding for a steady state velocity strengthening 
frictional regime $(a-b)>0$. Earthquakes occur when the velocity-weakening region of the fault exceeds a critical nucleation size that depends on the shear moduli of near-fault rocks, effective normal stress and frictional parameters (Rice, 1993; Rubin \& Ampuero, 2005). The evolution of the state variable is governed by the aging law:

$$
\frac{d \theta}{d t}=1-\frac{\dot{\delta} \theta}{L}
$$

Since the above formulation (Eq. 1) has singularity at slip rate $\dot{\delta}=0$, we use a regularized formulation for the shear strength, which is interpreted as a thermally activated creep model for the term $a \ln \left(\frac{\dot{\delta}}{\dot{\delta}_{0}}\right)$ in Eq. 1 (Rice \& Ben-Zion, 1996; Lapusta et al., 2000):

$$
T=a \bar{\sigma} \operatorname{arcsinh}\left[\frac{\dot{\delta}}{2 \dot{\delta_{o}}} e^{\frac{f_{o}+b \ln (\dot{\delta} \theta / L)}{a}}\right]
$$

We use a depth dependent profile for $(a-b)$ as inferred from granite samples in laboratory experiments (M. Blanpied et al., 1991; M. L. Blanpied et/al., 1995). The seismogenic zone is the velocity weakening region extending from a depth of $2 \mathrm{~km}$ to $15 \mathrm{~km}$. The rest of the fault is velocity strengthening and accommodates aseismic creep. The velocity strengthening region at the top $2 \mathrm{~km}$ of the fault is suggested by laboratory observations under low stresses (M. Blanpied et al., 1991). The effective normal stress is constant below the depth of $2 \mathrm{~km}$, since the increase in the lithostatic stress is accommodated by the pore fluid pressure at depth (Rice, 1993). The seismogenic zone is overstressed initially (Fig. 2b).

\subsection{Numerical Simulation of Fully Dynamic Earthquake Sequences}

We use a spectral element method to simulate dynamic ruptures and aseismic creep on the fault (Kaneko et al., 2011). Full inertial effects are considered during earthquake rupture and an adaptive time stepping technique is used to switch from interseismic to seismic events based on a threshold maximum slip velocity on the fault. This method is able to capture all four phases of the earthquake cycle including nucleation, rupture propagation, post seismic deformation, and interseismic creep. We implement Kaneko et al. (2011)'s algorithm in Julia (Bezanson et al., 2017) using a more efficient linear solver based on the Algebraic Multigrid scheme (Ruge \& Stüben, 1987) for the elliptic (interseismic) part of the earthquake sequence. This iterative technique uses a fixed number of iterations independent of the mesh size. In addition, we use the built-in multithreading feature of Julia, which enables us to achieve a CPU speed-up of $\sim 50$ times compared to the original code described in Kaneko et al. (2011).

\subsection{Theoretical Nucleation Estimates and choice of $L$}

In a two-dimensional continuum model, the theoretical estimate of earthquake nucleation for a mode III crack based on energy balance is given by (Rubin \& Ampuero, 2005):

$$
h^{*}=\frac{2}{\pi} \frac{\mu L b}{\bar{\sigma}(b-a)^{2}}
$$

where $a, b$, and $L$ are the rate and state friction parameters, $\mu$ is the shear modulus of the near source region and $\bar{\sigma}$ is the effective normal stress. Using $L=8 \mathrm{~mm}$ leads to a nucleation size of $3.9 \mathrm{~km}$ in a homogeneous medium. As the nucleation size is proportional to the rigidity of the near-source medium (Rubin \& Ampuero, 2005; Kaneko et al., 2011), it is reduced by a factor of $\sim 3$ in a damaged medium with a shear wave velocity reduction of $40 \%$ (Huang, 2018). The theoretical estimate of the nucleation size in a layered medium for a mode III rupture is derived by Kaneko et al. (2011) using linear stability analysis:

$$
h_{\text {lay }}^{*} \tanh \left[2 H \frac{\gamma}{h_{\text {lay }}^{*}}+\operatorname{arctanh}\left(\frac{\mu_{D}}{\mu}\right)\right]=h_{\text {hom }}^{*}
$$



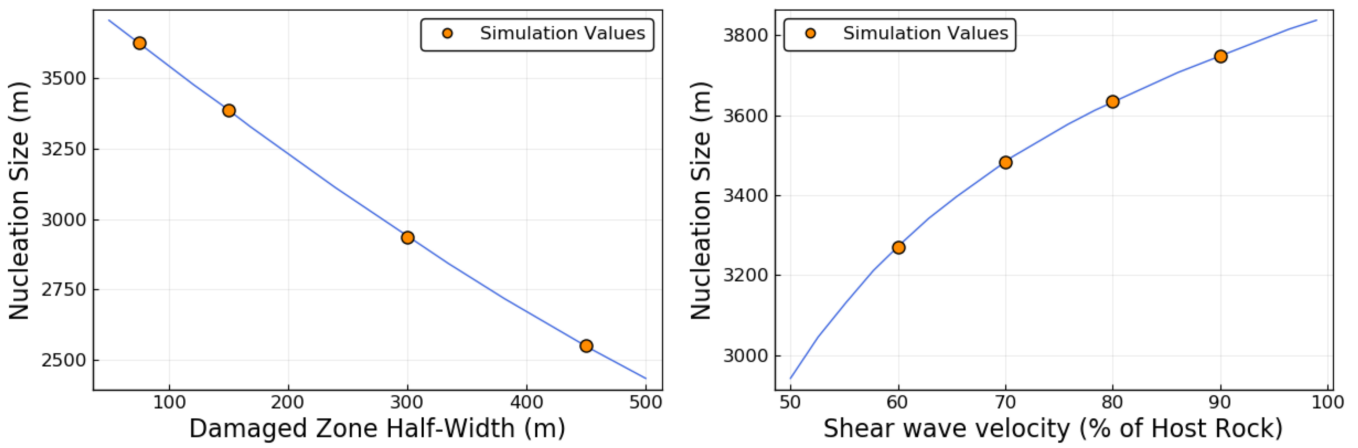

Figure 3. Variation of theoretical nucleation sizes in a layered medium. The left figure shows the variation due to fault damage zone widths, and the right figure shows the variation due to shear wave velocity. The orange dots show the theoretical nucleation sizes for the parameters chosen in our simulations.

where $\mu$ and $\mu_{D}$ are the rigidity of the host rock and the layer respectively, $\gamma(=\pi / 4)$ is an empirical parameter dependent on the geometry, and $\mathrm{H}$ is the thickness of the layered medium. The parameter choice of width and shear wave velocity contrast and their corresponding nucleation sizes are shown in Fig. 3. A smaller nucleation size would allow smaller earthquakes to nucleate successfully, therefore incorporating a wider range of magnitudes. We use 5 Gauss-Lobatto-Legendre nodes inside each spectral element, such that the average node spacing is $20 \mathrm{~m}$. For a well resolved simulation, the cohesive zone size (Day et al., 2005; Kaneko et al., 2008) should contain at least 3 node points. Based on the frictional parameters and rigidity of fault damage zone, the cohesive zone size in our models is $\sim 120 \mathrm{~m}$ and encompasses sufficient nodes. We demonstrate the convergence of our model with respect to different node spacings in Appendix A.

\section{Results}

Our results show that the presence of the fault damage zone promotes complexity in the earthquake slip distribution and variability in their magnitudes, especially for large rigidity contrast between the fault damage zone and the host rock. Given the friction parameters and initial stress conditions in our simulations (Fig. 2b), the homogeneous medium hosts periodic earthquakes with exactly the same hypocenter locations and magnitudes, whereas the fault surrounded by a fault damage zone shows a more complex slip distribution with variable earthquake sizes and hypocenter locations through multiple earthquake cycles (Fig. 4). We also observe ruptures with multiple slip pulses and more complex slip distribution in the flower structure scenario (Fig. 4d, Fig. 5b).

Previous dynamic rupture simulations show that fault zone wave reflections can induce pulse-like ruptures (Harris \& Day, 1997; Huang \& Ampuero, 2011; Huang et al., 2014). We observe the imprint of these wave reflections in the spatiotemporal slip rate evolution of fault damage zone simulations (Fig. 5). These Slip pulses become a dominant feature during earthquake rupture as the waves are reflected from the damage zone boundaries in our earthquake cycle simulations. Similar pulse-like ruptures are also observed in homogeneous earthquake cycle simulations for specific sets of heterogeneous friction parameters and fault asperity dimensions (Michel et al., 2017; Barbot, 2019). Our results suggest that stress heterogeneities generated by slip pulses due to seismic wave reflections are primarily responsible for the complexities in accumulated slip and variation in hypocenter distributions. 
(a) Model I: Homogeneous Medium

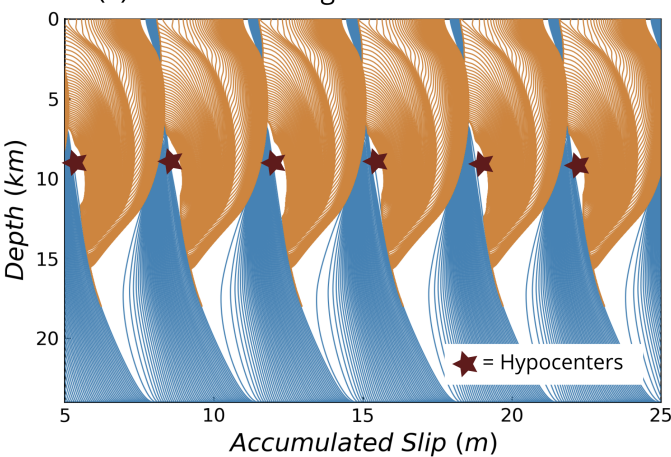

(c) Model III: Shallower (10 km) damage zone

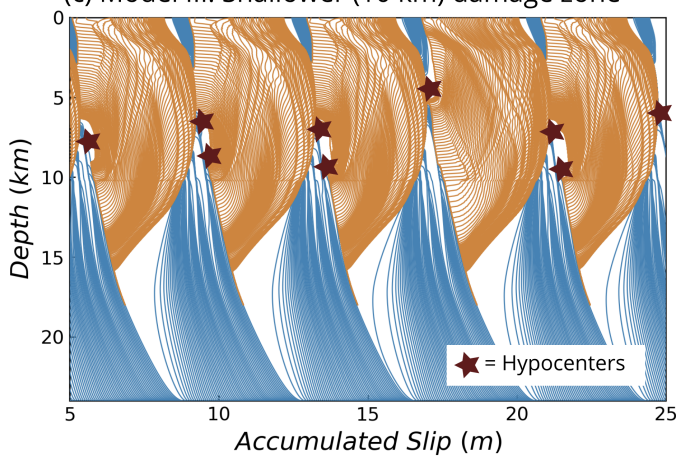

(b) Model II: Damage zone throughout the domain

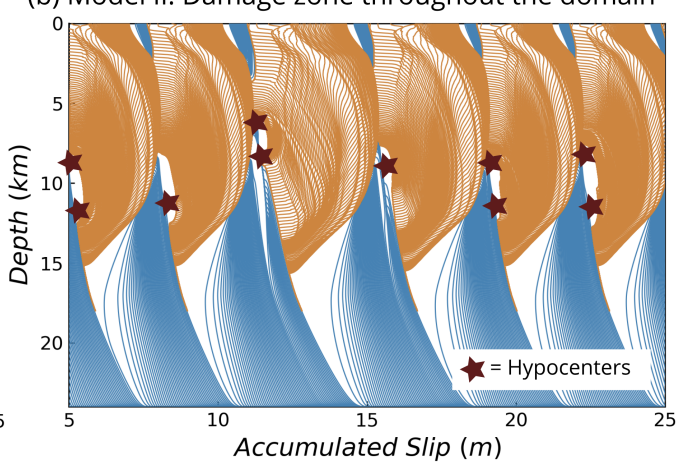

(d) Model IV: 2-D flower structure

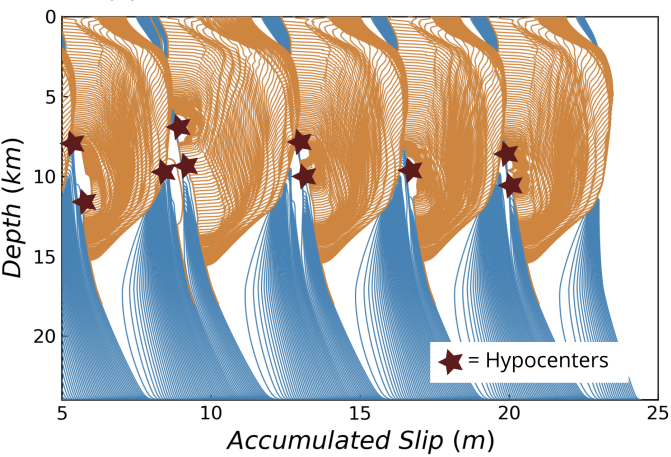

Figure 4. Cumulative slip contours with hypocenters shown as red stars. Multiple hypocenters close to each other represent smaller $(\mathrm{Mw} \sim 3)$ and larger $(\mathrm{Mw} \sim 7)$ earthquakes. The orange lines are plotted every $0.1 \mathrm{~s}$ during an earthquake and the blue lines are plotted every $2 \mathrm{yr}$ during the interseismic period. Four different models include (a) homogeneous medium, (b) fault damage zone extending throughout the domain, (c) fault damage zone truncated at a shallower depth of $10 \mathrm{~km}$, and (d) 2D flower structure. The half-width of the damage zone is $150 \mathrm{~m}$ in (b) and (c) and the shear wave velocity reduction is $40 \%$ of host rock. The inner damage zone in (d) is $150 \mathrm{~m}$ wide with $40 \%$ shear wave velocity reduction and the outer damage zone is $1.5 \mathrm{~km}$ wide with $20 \%$ shear wave velocity reduction.

We compute the moment magnitudes of simulated earthquakes to investigate the relation between the magnitudes and cumulative number of earthquakes. The start and end of a rupture is definedd based on a threshold slip velocity of $0.001 \mathrm{~m} \mathrm{~s}^{-1}$. The seismic moment is calculated as the product of the elastic shear modulus $(\mu)$, the coseismic slip $(D)$ integrated along the depth, and the rupture area. The rupture length $(L)$ is defined as the part of the fault where slip is greater than $1 \%$ of the maximum coseismic slip during a certain earthquake. Since our simulation is two-dimensional, we assume the rupture width $(W)$ is the same as the rupture length. The seismic moment $\left(M_{o}\right)$ is defined as:

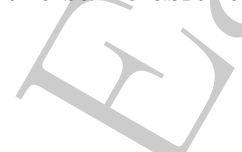

$$
M_{o}=\mu(L . W) D=\int d L \int \mu d L \cdot D(L)
$$

The moment magnitude is computed using Kanamori's (1975) scaling relation: $M w=$ $2 / 3 \log M_{o}-10.7$, where $M_{o}$ is the seismic moment.

In our simulations, the model with homogeneous medium hosts one large earthquake every $\sim 100$ years. The recurrence intervals and magnitude of the earthquakes are also fairly uniform throughout the seismic cycle. In the presence of the fault damage zone, 
(a)

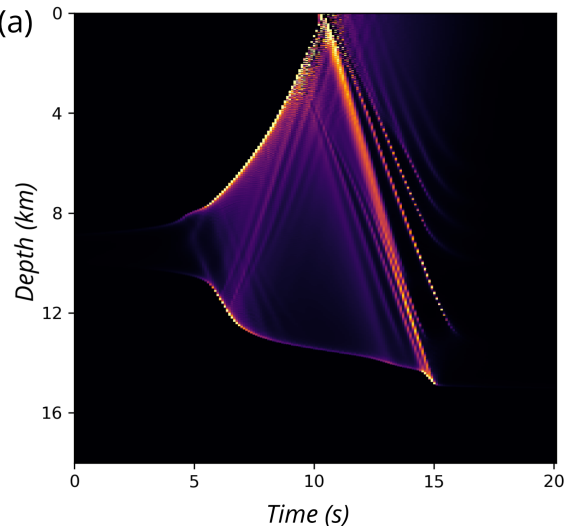

(c)

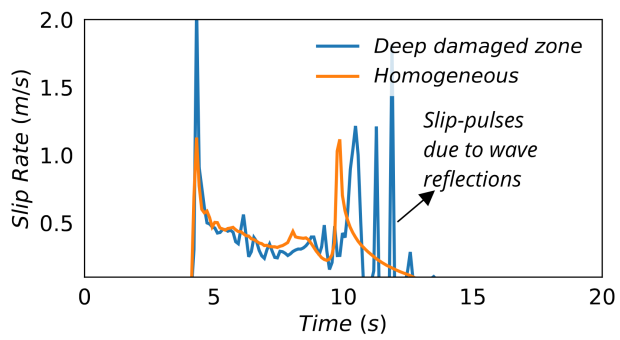

(b)

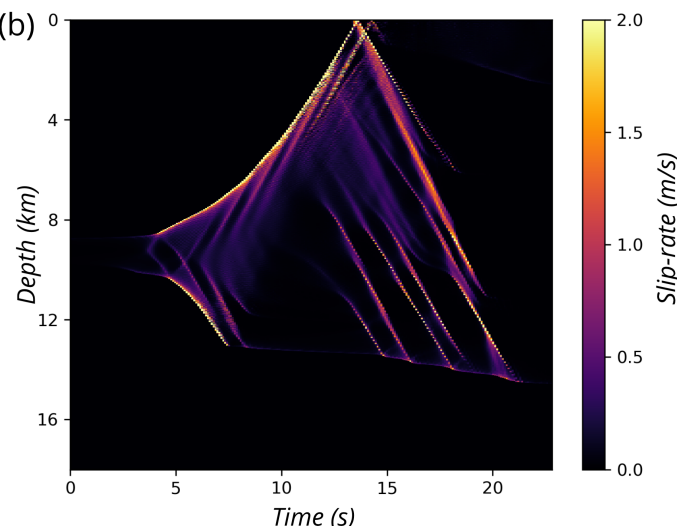

(d)

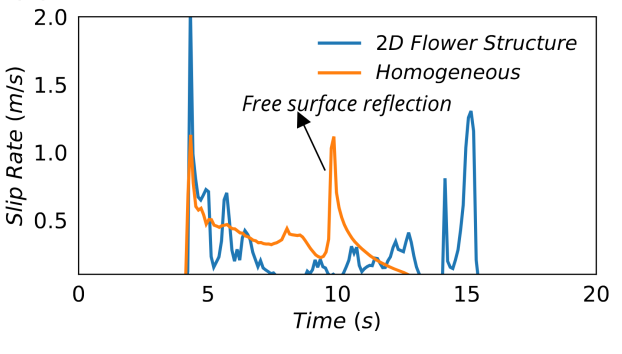

Figure 5. Spatiotemporal slip rate evolution demonstrating dynamic wave reflections for (a) fault damage zone extending throughout the domain, and (b) trapezoid shaped nested fault damage zone. (c) and (d) show the slip rate at a depth of $7 \mathrm{~km}$ for (a) and (b) respectively as compared to a homogeneous medium. The ruptures begin as crack but transition to pulses due to the wave reflections.

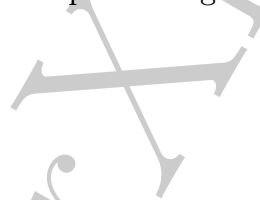

we observe more complex slip history with varying earthquake magnitudes and hypocenter locations. To further understand the simulated earthquake catalog, we investigate the number of earthquakes for each mágnitude range (i.e., magnitude-frequency distribution). We combine the magnitudes for all the fault zone simulations in order to emulate a natural setting where there are multiple faults with varying fault damage zone properties and show their cumulative magnitude frequency distribution in Fig. 6a. We observe a decrease in the number of earthquakes as the magnitude increases from 3 to 4.5 , after which the number of earthquakes stagnates for intermediate magnitudes of 4.5 to 6 . Finally we see a sharp decrease in the number of earthquakes for the largest earthquakes. This combined magnitude-frequency distribution is different from the Gutenberg-Richter distribution.

To understand the gap in the intermediate magnitude earthquakes, we examine the envelope of the coseismic slip distributions representing the rupture area for all the simulated earthquakes (Fig. 6b). The rupture areas of smaller earthquakes are confined within the depth range of $3 \mathrm{~km}$ to $11 \mathrm{~km}$ (Fig. 6b). The rupture area and final slip for these subsurface events are $\sim 10$ times smaller than those of the surface-rupturing events. Therefore there is two orders of gap in the moment magnitudes between the small and large events. Since the effective normal stress and hence the fault strength is low at depths shallower than $3 \mathrm{~km}$, it is harder to stop dynamic ruptures once they reach this shallow depth. When the rupture breaks through the free surface, the magnitude of the earthquakes tend to be much larger, which may explain the lack of intermediate magnitude earthquakes. Another potential reason is that there is no along-strike rupture termination in our $2 \mathrm{D}$ models. Generating a Guternberg-Richter type earthquake catalogue may require multiple faults 
with varying spatial dimensions, additional frictional or material heterogeneities, and/or along-strike termination of spontaneous ruptures.

(a)

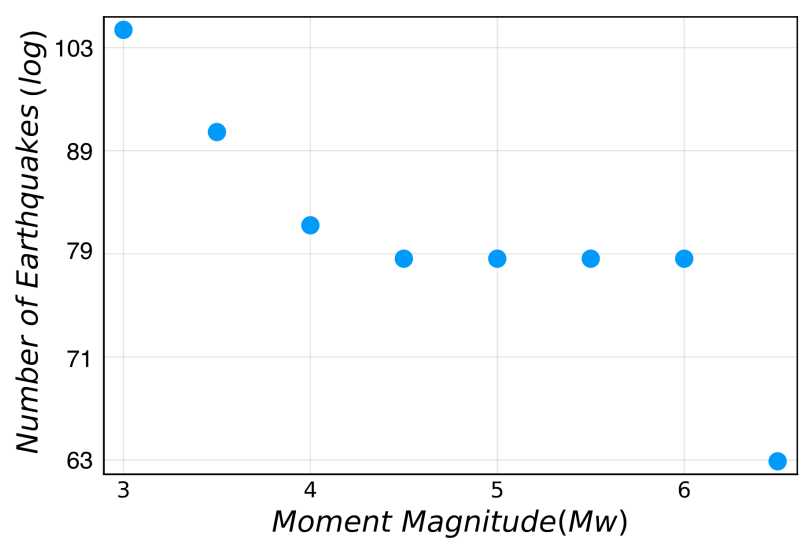

(b)

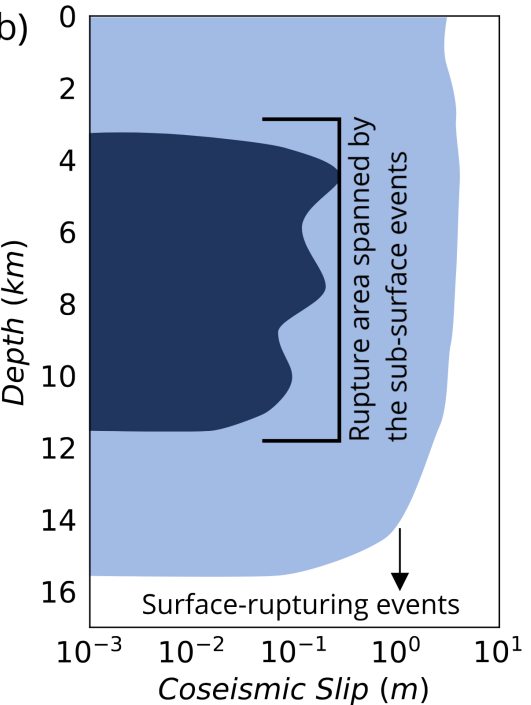

\subsection{Variability in Earthquake Hypocenters}

Earthquakes on crustal strike-slip faults tend to occur within the top $15 \mathrm{~km}$ to $20 \mathrm{~km}$ of the crust, known as the seismogenic zone. However, these earthquakes are not uniform along depth, and are more correlated with the shallow crustal structure (Marone \& Scholz, 1988). Mai et al. (2005) have performed Kolmogorov-Smirnov tests on a database of finite-source inversions and showed that the uniformity of hypocenters along depth can be statistically rejected, especially for strike-slip faults. Other studies (Marone \& Scholz, 1988; Hauksson \& Meier, 2019) have shown that the depth distribution of earthquake hypocenters may be more bimodal, with strong clustering of earthquakes at shallow $(\sim 5 \mathrm{~km})$ and deeper $(\sim 15 \mathrm{~km})$ depths. Such a bimodal distribution has also been observed in thrust fault settings (Dal Zilio, 2020). Shallow seismicity is usually interpreted as short-term strain transients or changes in the frictional and rheological properties of rocks along depth. The abrupt decrease in deeper seismicity $(\leq 15 \mathrm{~km})$ is attributed to the thermo-mechanical behavior of rocks at these depths. We provide an alternate explanation for the bimodal distribution of seismicity along strike-slip faults based on the geometrical extent of fault damage zones, wherein the structural boundary of the fault damage zone produces additional stress concentration that promotes earthquake nucleation near the boundary. Our results also suggest that frictional and rheological effects may be a dominant mechanism for hypocenter variability when the damaged structure is shallower than $8 \mathrm{~km}$.

The depth distributions of earthquake hypocenters for various fault zone depths, widths and velocity contrasts are shown in Fig. 7. In contrast to the homogeneous medium, the 
(a). Depth variation of fault damaged zones
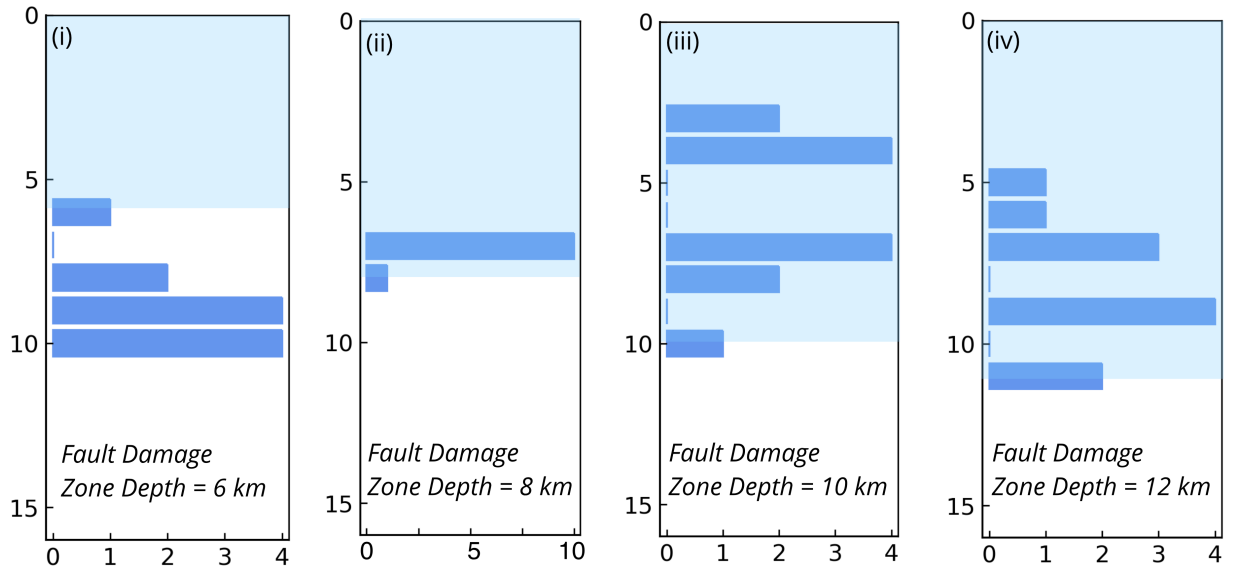

(b). Width variation of fault damaged zones
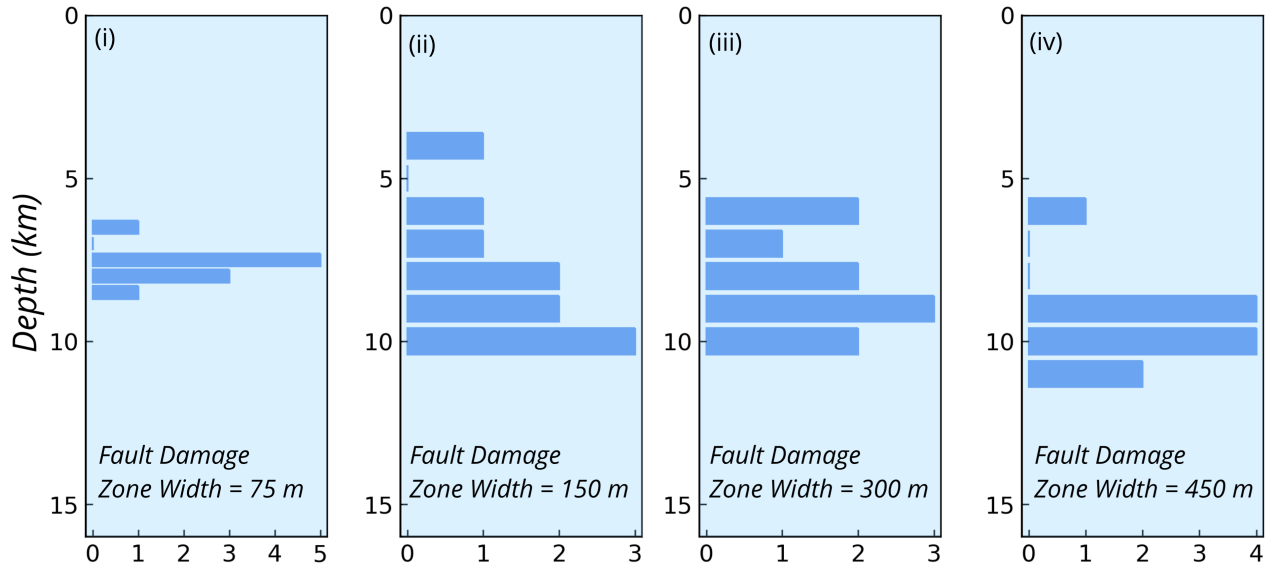

(c). Shear wave velocity variation of fault damaged zones (as percentage of host rock)
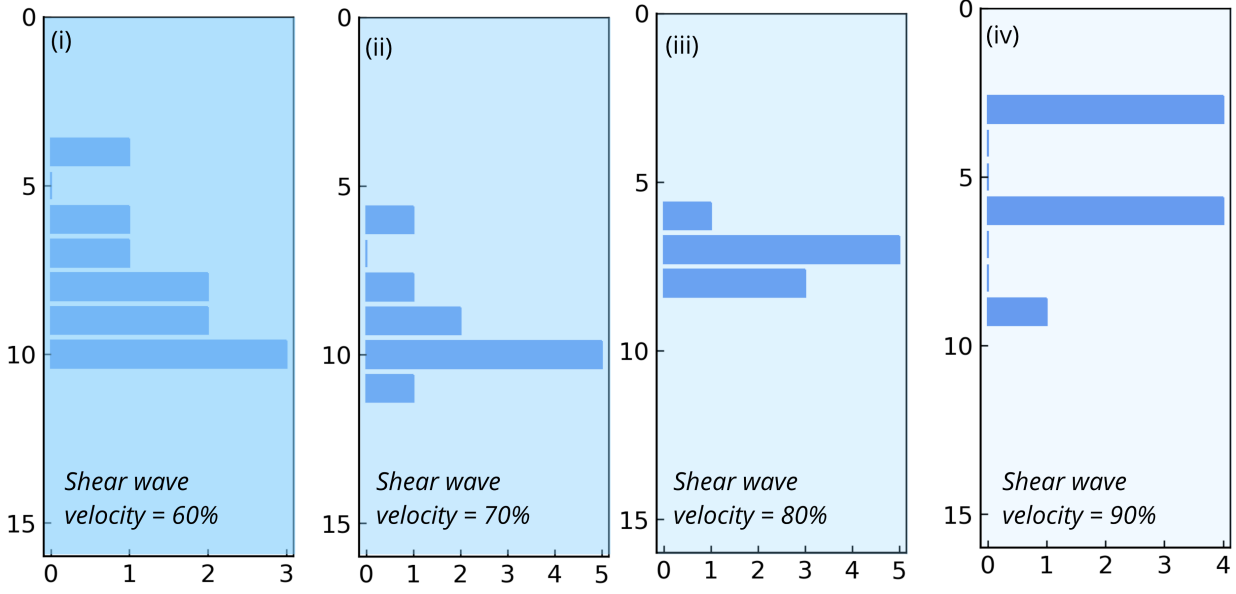

Number of Earthquakes

Figure 7. Earthquake hypocenter distribution for simulations with varying (a) fault damage zone depths, (b) widths, and (c) shear wave velocity contrasts. The shaded region shows the depth extent of damage zone and the intensity of shading shows the shear wave velocity contrast. 
hypocenter locations vary considerably for the fault zone simulations, and the depth extent of the fault damage zone has a pronounced effect on the hypocenter location. As demonstrated by Fig. 7a, the maximum variability in hypocenter locations is observed when the fault damage zone extends to the earthquake nucleation sites. As the fault zone becomes deeper, we see a systematic downward shift in the average hypocenter location, which saturates for a very deep fault zone extending throughout the seismogenic zone. We attribute this variability to the sharp material discontinuity between the fault damage zone and the host rock where shear stress changes tend to be concentrated (Bonafede et al., 2002; Rybicki \& Yamashita, 2002), resulting a number of earthquakes nucleating near this interface. For the same depth below the shallower fault zone, the deeper fault zone leads to a smaller nucleation size due to the reduction in elastic shear modulus, thus allowing earthquakes to nucleate at a deeper location as the fault is loaded from below. However, when the damage zone is very shallow, in the order of $\sim 6 \mathrm{~km}$ depth (Fig. 7a), most of the earthquakes nucleate below the damage zone. This suggests that the interplay between the earthquake nucleation site and damage zone boundary is an important factor influencing earthquake hypocenter locations. Despite additional stress concentration at the fault damage zone boundary, fault loading conditions and frictional boundary have a dominant effect on earthquake hypocenters for very shallow fault zone. But as the fault damage zone penetrates to the nucleation site, the fault zone effects become more critical in determining the depth distribution of seismicity. In other words, the seismicity distribution is influenced by both the material and frictional boundaries.

In fault damage zones extending throughout seismogenic depths, the increase of damage zone width also leads to an increase in the average hypocenter depths (Fig. 7b). This is consistent with the idea that the nucleation size is reduced as the width increases, which should lead to a downward shift in earthquake hypocenters when the fault loaded from below. The hypocenter locations also tend to be deeper for a higher shear wave velocity contrast, again due to a smaller nucleation size (Fig. 7c).

Our simulations highlight the variable depth distribution of earthquake hypocenters on strike-slip faults. In certain cases, a shallow fault damage zone exhibits more bimodal distribution of hypocenters (Fig. 7a), whereas deeper fault damage zones tend to exhibit more unimodal distribution (Fig. 7b). We also see a bimodal distribution when the shear wave velocity contrast is very low (Fig. 7c), which can be attributed to frictional stress concentrations. We show the hypocenter distributions from two representative simulations of a shallow and a deep fault damage zone against various observations (Fig. 8a), wherein the shallower damage zone shows a more bimodal distribution as compared to a deeper damage zone (Fig. 8b). It is pertinent to note that most of the observations of seismicity depth distribution is limited to small earthquakes, because we do not have enough record of large earthquakes along single faults. Nevertheless, we are qualitatively able to compare the simulated earthquake hypocenter locations with the observed hypocenter locations.

\subsection{Evolution of Peak Slip Rate and Fault Shear Stresses}

We show the peak slip rate evolution for our simulations in Fig. 9. A homogeneous simulation shows large recurring earthquakes, whereas smaller events emerge in a damaged medium, caused by the interplay between the fault damage zone boundary and the nucleation along the fault. In addition, we observe multiple 'slow events' in the presence of the fault damage zone that do not grow to fully dynamic earthquakes. The complexities in the number of these 'slow events' are elevated for a shallow fault damage zone extending to the nucleation site (Fig. 9c). The flower structure shows a more complex peak slip rate function (Fig. 9d) despite having fewer slow events because the inner damage zone extends deep within the seismogenic zone. These slow events in our models occur at $\sim 10 \mathrm{~km}$ depth (Fig. $4 \mathrm{~b}, \mathrm{~d}$ ), close to the nucleation site and also close to the damage boundary in the case of shallower fault damage zone (Fig. 4c). They can be interpreted as accelerations in the slip rate that cannot grow to fully dynamic earthquakes because the stresses are not 


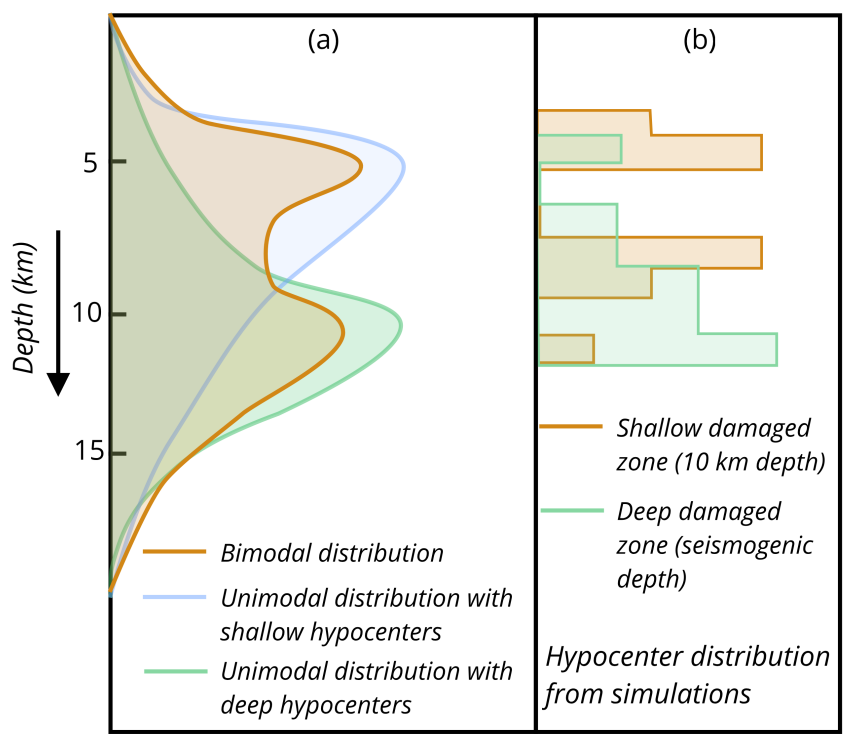

Number of Earthquakes

Figure 8. (a). Observed seismicity distribution along strike-slip faults. We show bimodal distribution (Marone \& Scholz, 1988; Mai et al., 2005; Hauksson \& Meier, 2019), unimodal distribution with shallow hypocenters (Powers \& Jordan, 2010; Kim et al., 2016), and unimodal distribution with deep hypocenters (Hauksson \& Meier, 2019). (b) Simulated hypocenter distribution for a shallow and a deep damage zone.

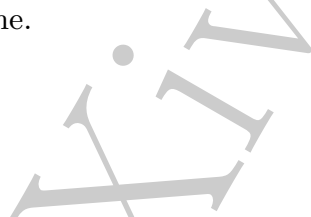

large enough to reach the dynamic regime, i.e., a failed nucleation. Slow slip events with back-propagating pulses have previously been observed in low-velocity fault zone simulations under the quasidynamic approximation Idini and Ampuero (2020)(EarthArXiv preprint). These events are modeled using additional frictional strengthening. We observe a combination of slow events and dynamic/ruptures in the velocity weakening regime. Our results imply that the geometry of the damaged medium can cause additional source complexities that are similar to seismic observations. We infer that a mature fault zone is more likely to exhibit slow events compared to immature fault zones in strike-slip tectonic settings.

In order to understand the mechanism underlying the variability of earthquake hypocenter locations and the scale of stress heterogeneities, we show the temporal evolution of fault shear stresses for different types of fault zones. Fig. 10 shows the shear stress evolution for the largest earthquake in homogeneous medium $(L=8 \mathrm{~mm})$, a deeper fault damage zone, a shallow fault/damage zone, and the 2D flower structure, respectively. Ruptures in the fault zone undergo a transition from cracks to pulses predominantly after the waves are reflected from the fault damage zone boundaries (Fig. 5a), while the homogeneous simulations maintain crack-like ruptures. In addition to the observed reduction in nucleation sizes, shear stress heterogeneities emerge during the nucleation phase in the damage zone simulations (Fig. 10b), whereas they are absent in homogeneous medium (Fig. 10a). The interference of multiple stress peaks very close to the nucleation site are responsible for the variability in earthquake hypocenter locations and sizes in the fault zone simulations. The emergence of smaller earthquakes $(\mathrm{Mw} \sim 3.0)$ and the slow events are prominent when a fault damage zone extends to the nucleation site of the earthquakes. Although earthquake rupture velocities are slower in the fault damage zone, the stress peak amplitudes are larger than the homogeneous medium. Overall, the two key effects of the fault damage zoned in 
(a) Model I: Homogeneous Medium

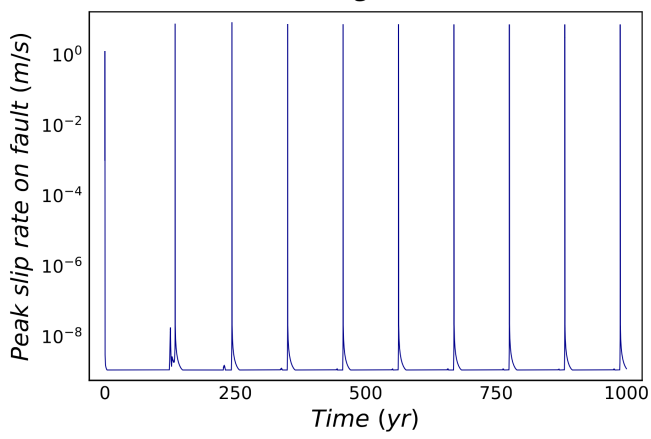

(c) Model III: Shallower (10 km) damage zone

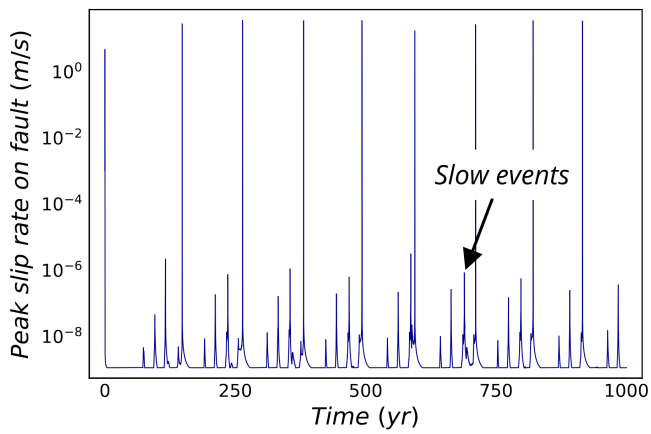

(b) Model II: Damage zone throughout the domain

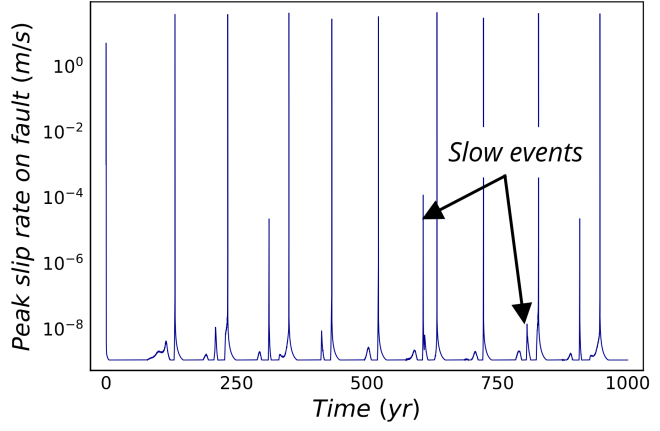

(d) Model IV: 2-D flower structure

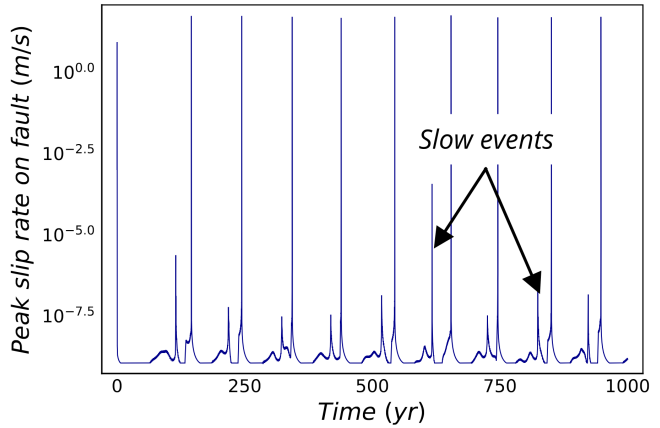

Figure 9. Peak slip rate function for (a) homogeneous medium, (b) deep fault damage zone, (c) shallow fault damage zone, (d) two-dimensional flower structure.

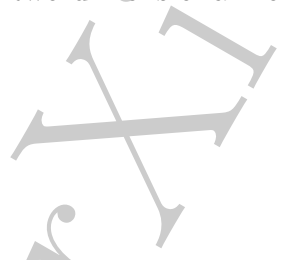

fully dynamic earthquake sequences are: (a) multiple stress peaks near the nucleation site, (b) small-scale stress heterogeneities due to dynamic wave reflections.

\subsection{Effects of Reduced Nucleation Sizes in Homogeneous Medium}

The theoretical nucleation size of a mode III rupture is directly proportional to the rigidity of the medium. Since smaller nucleation sizes also tend to give rise to complexities in earthquake cycles (Lapusta \& Rice, 2003), it is imperative to isolate the effects of reduced nucleation size from the effects of dynamic wave reflections and stress heterogeneities due to the fault damage zones. In this section, we analyze a simulation where the entire medium is damaged and has a $40 \%$ shear wave velocity reduction compared to the homogeneous medium in previous simulations. The cumulative slip profile and the shear stress evolution (Fig. 11) show a clear reduction in nucleation size when compared to the undamaged homogeneous medium (Fig. 4a) as expected from theoretical predictions. Furthermore, we see a downward shift in earthquake hypocenter locations, which is also an expected phenomenon for mode III earthquake cycles due to the loading conditions (Lapusta \& Rice, 2003). Another effect is the increase in recurrence intervals of the large earthquakes, which can be attributed to a reduced shear modulus in the medium (Cattania, 2019). Despite these differences, we do not observe complexities such as variations in hypocenter locations or earthquake sizes as observed in fault zone simulations. Therefore, these complexities can be attributed to dynamic wave reflections and stress heterogeneities induced by the complex nucleation phase (Fig. $10 \mathrm{~b}, \mathrm{c}, \mathrm{d}$ ). 

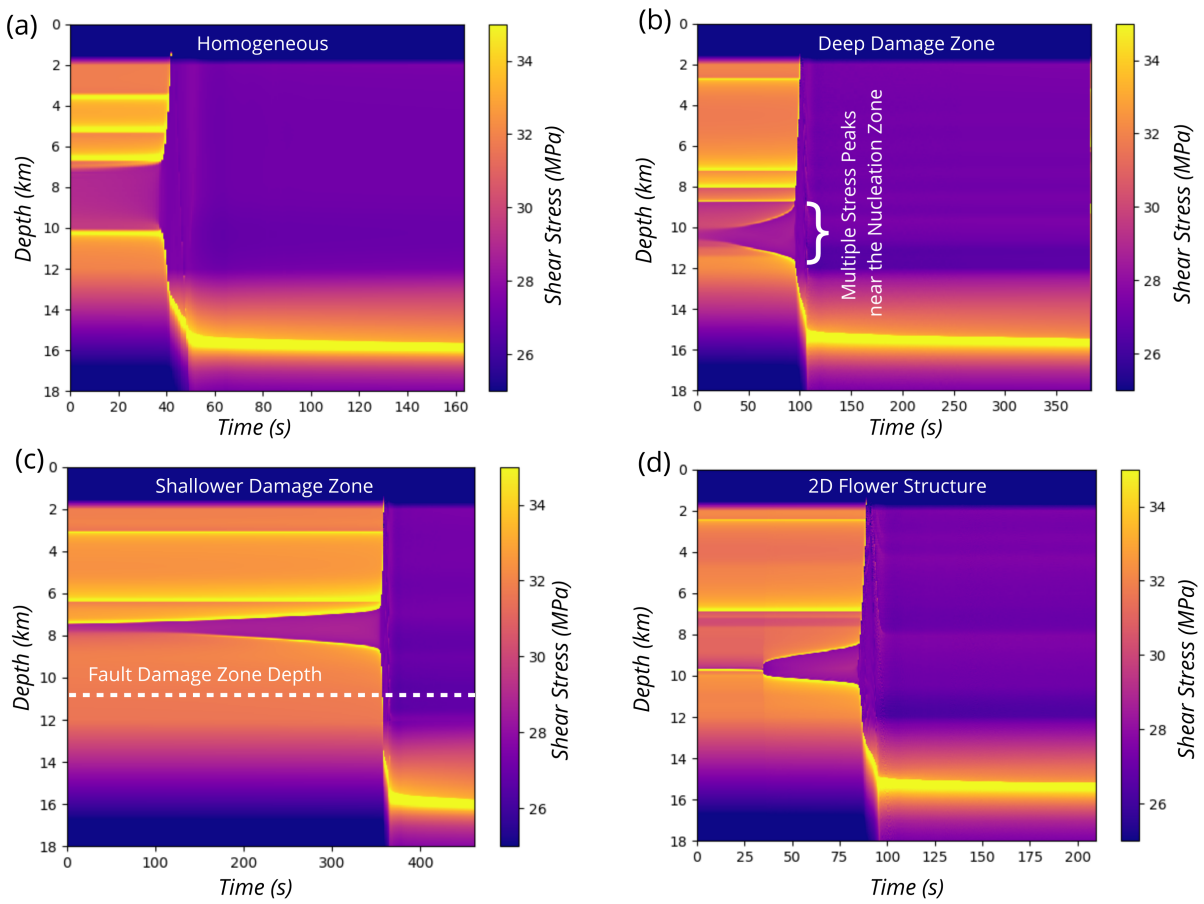

Figure 10. Shear stress evolution of a single earthquake including the nucleation phase shown along the fault for (a) homogeneous medium, (b) deep fault zone, (c) shallower fault zone, (d) two-dimensional flower structure.
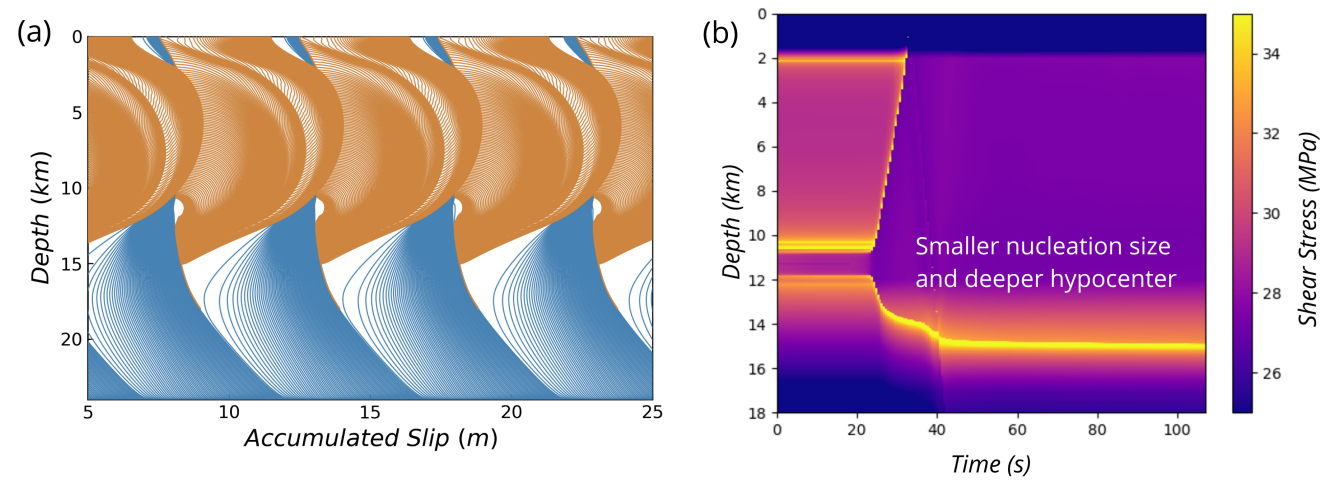

Figure 11. (a) Cumulative slip evolution with the orange lines plotted every $0.5 \mathrm{~s}$ and blue lines plotted every 2 yr. (b) Shear stress evolution for one earthquake when the entire medium is damaged. The shear wave velocity is $60 \%$ that of undamaged medium (compare Fig. 4a), which would effectively reduce the nucleation size by $66 \%$. We do not see variability in hypocenter locations or earthquake magnitude.

\section{Discussion and Conclusions}

We present fully dynamic earthquake cycle models that incorporate near-fault material heterogeneities represented by a fault damage zone. We show that the fault zone waves can 
lead to earthquakes with variable magnitudes and hypocenter locations. The depth distribution of earthquake hypocenters is strongly affected by the fault damage zone depth, with shallower fault zones favoring shallower hypocenters. We also see a bimodal depth distribution of earthquake hypocenters in shallow damage zones and a more unimodal distribution in deeper damage zones. The variable nucleation locations originate from the interaction between stress heterogeneity induced by dynamic fault zone waves and the rate and state fault. In the shallow fault zone, the stress peaks are concentrated near the bottom of the fault damage zone and directly correlated with the earthquake nucleation locations, whereas the complex nucleation phase is absent in the homogeneous media.

Previous static and quasi-dynamic simulations have shown that perturbations in shear and normal stress can give rise to complex seismicity (Ben-Zion, 2001; Perfettini et al., 2003). Furthermore, observations and numerical experiments suggest that the tectonic stresses on real faults are spatially heterogeneous (Townend \& Zoback, 2000; Rivera \& Kanamori, 2002 ), implying that the stress amplitudes are not smooth but oscillatory over space. The emergence of persistent slip pulses after initial few seconds of rupture propagation contribute to stress heterogeneity in our simulations. Another key observation is the emergence of smaller, slower events in the damaged medium that do not grow to dynamic earthquakes. These slow events are more prominent in the shallow fault zones where the depth of the fault damage zone intersects the nucleation zone but does not extend deeper to the seismogenic zone. This suggests that the material heterogeneities strongly influence the nucleation phase in addition to generating dynamic reflected waves. The emergence of these slow events in our fault damage zone simulations could potentially explain why we do not observe slow slip events in young and immature fault zones.

We find that the shape and properties of damage zone can affect the stress distribution and significantly contribute to complex seismicity even without smaller-scale frictional heterogeneities along fault. Earthquake magnitudes show significant variability when compared to a homogeneous medium, but the log-linearity of the magnitude-frequency distribution is difficult to reproduce due to the limited number of earthquakes generated in the simulations. Observations in regional and global earthquake catalogues generally show a log-linear decay of magnitude with increasing number of earthquakes, in agreement with the GutenbergRichter distribution. However, large earthquakes along individual faults or fault sections deviate from this behavior, showing a relatively elevated number of 'characteristic earthquakes' (Schwartz \& Coppersmith, 1984; Wesnousky, 1994; Parsons et al., 2018) that follow a gaussian distribution in addition to smaller earthquakes that follow the Gutenberg-Richter distribution. This characteristic distribution is used as a basis for rupture forecast models, e.g., (Field et al., 2017). We have combined the earthquakes from multiple simulations to emulate a regional catalogue where we may have multiple faults with different fault zone characteristics, but we ignore the interactions between these faults. In order to reproduce a Gutenberg-Richter distribution, more complexities in the model are required. The question still remains whether/we need only material heterogeneities, or additional frictional and stress heterogeneities in combination to emulate the Gutenberg-Richter behavior. The current model is an idealized approximation of the material effects of fault damage zones with small fractures. More realistic approximations would include the incorporation of viscoelastic and plasticity effects (Allison \& Dunham, 2018; Erickson et al., 2017) and variable pore pressure effects with depth. Furthermore, complexities in the frictional parameters $(a-b)$ and $L$ are limited to standard values that remain constant throughout simulating time. Despite these approximations, our models provide a physical description of the effects of material heterogeneities on the long-term behavior of strike-slip faults.

Our future work will be directed towards understanding the effect of fault damage zone evolution through multiple seismic cycles. Paleoseismic studies of large strike-slip earthquakes, limited to the past 1000-1200 years, suggest that the recurrence of large events is non-uniform, possibly even chaotic, with large gap in seismic activity followed by multiple seismic episodes (Grant \& Sieh, 1992; Seitz et al., 1997; Fumal et al., 2002; Toké et al., 
2006). A time-dependent stressing history, possibly driven by the evolution of the fault damage zone through multiple seismic episodes and aseismic creep, may better explain the observed non-uniform recurrence intervals along mature faults. Previous experiments and observations (Peng \& Ben-Zion, 2006; Stanchits et al., 2006) have shown that the damage can be enhanced during seismic episodes and be healed during interseismic periods. The amount and localization of damage depends on the earthquake sizes, the interseismic duration for which the fault is allowed to heal, and recurrence intervals of large earthquakes (Vidale \& Li, 2003; Yang, 2015). Incorporating the evolution of fault damage zone would provide more realistic outlook on long-term structural evolution and source characteristics of mature strike-slip faults.

\section{Appendix A Numerical Convergence in the Simulations}

We perform numerical convergence tests for the simulations with a narrow fault damage zone extending throughout the model domain. The half-width of the fault damage zone is $150 \mathrm{~m}$, and the shear wave velocity reduction is $40 \%$. We use an average node-spacing of $10 \mathrm{~m}, 15 \mathrm{~m}, 20 \mathrm{~m}$ and $40 \mathrm{~m}$. The comparison between the peak slip rate and the differential slip for a large earthquake is shown in Fig. A1. The comparison of peak slip rate for simulations with different node spacings demonstrates that the onset of earthquakes are the same for the different node spacings. Furthermore, Fig. A1 b shows that the differential slip for different node spacings are the same, implying that the earthquake size is independent of mesh size. The shape of the differential slip shown in the inset zoom figure (Fig. A1 b) suggests all the features are not preserved for an average node spacing of $40 \mathrm{~m}$, but they are preserved for all the other node spacings. Based on this convergence study, we have chosen an average node spacing of $20 \mathrm{~m}$ for our study.
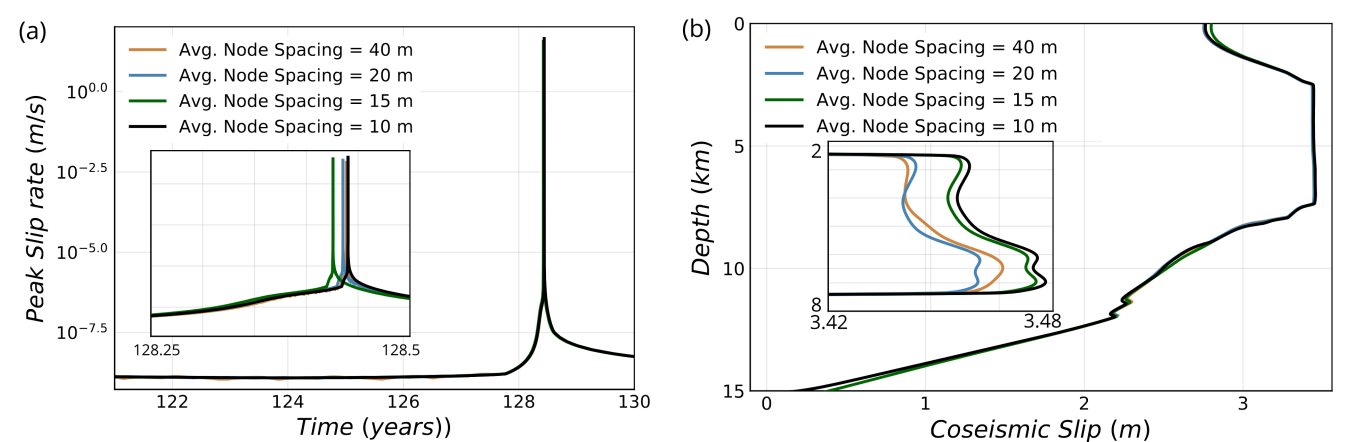

Figure A1. (a) Peak slip rate shown for multiple node spacings, (b) Differential slip of one earthquake shown for multiple node spacings.

\section{Acknowledgments}

This study was supported by the University of Michigan. We thank the editor - - - and reviewers - - - for their helpful comments. P.T thanks Daning Huang for help with the numerical procedure for implementing the Algebraic Multigrid for quasi-static solver. The code used to perform the numerical simulations is available on github: https:// github.com/thehalfspace/eqcycle. GMT (Wessel et al., 2013) was used to create some figures. 


\section{References}

Abdelmeguid, M., Ma, X., \& Elbanna, A. (2019). A novel hybrid finite element-spectral boundary integral scheme for modeling earthquake cycles: Application to rate and state faults with low-velocity zones. Journal of Geophysical Research: Solid Earth.

Albertini, G., \& Kammer, D. S. (2017). Off-fault heterogeneities promote supershear transition of dynamic mode ii cracks. Journal of Geophysical Research: Solid Earth, 122(8), 6625-6641.

Allison, K. L., \& Dunham, E. M. (2018). Earthquake cycle simulations with rate-and-state friction and power-law viscoelasticity. Tectonophysics, 733, 232-256.

Barbot, S. (2019). Slow-slip, slow earthquakes, period-two cycles, full and partial ruptures, and deterministic chaos in a single asperity fault. Tectonophysics, $768,228171$.

Barbot, S., Lapusta, N., \& Avouac, J.-P. (2012). Under the hood of the earthquake machine: Toward predictive modeling of the seismic cycle. Science, 336(6082), 707710. Retrieved from http://science.sciencemag.org/content/336/6082/707 doi: 10.1126/science. 1218796

Ben-Zion, Y. (2001). Dynamic ruptures in recent models of earthquake faults. Journal of the Mechanics and Physics of Solids, 49(9), 2209-2244.

Bezanson, J., Edelman, A., Karpinski, S., \& Shah, V.B. (2017). Julia: A fresh approach to numerical computing. SIAM review, 59(1), 65-98.

Blanpied, M., Lockner, D., \& Byerlee, J. (1991). Fault/stability inferred from granite sliding experiments at hydrothermal conditions. Geophysical Research Letters, 18(4), 609-612.

Blanpied, M. L., Lockner, D. A., \& Byerlee, J. D. (1995). Frictional slip of granite at hydrothermal conditions. Journal of Geophysical Research: Solid Earth, 100(B7), $13045-13064$.

Bonafede, M., Parenti, B., \& Rivalta, E. (2002). On strike-slip faulting in layered media. Geophysical Journal International, 149(3), 698-723.

Caine, J. S., Evans, J. P., \& Forster, C. B. (1996). Fault zone architecture and permeability structure. Geology, 24(11), 1025-1028.

Carlson, J. M., \& Langer, J. (1989). Properties of earthquakes generated by fault dynamics. Physical Review Letters, 62(22), 2632.

Cattania, C. (2019). Complex earthquake sequences on simple faults. Geophysical Research Letters, 46(17-18), 10384-10393.

Chester, F., \& Logan, J. M. (1986). Implications for mechanical properties of brittle faults from observations of the punchbowl fault zone, california. Pure and applied geophysics, 124 (1-2), 79-106.

Chester, F. M., Evans, J. P., \& Biegel, R. L. (1993). Internal structure and weakening mechanisms of the san andreas fault. Journal of Geophysical Research: Solid Earth, 98(B1), 771-786.

Cochard, A., \& Madariaga, R. (1996). Complexity of seismicity due to highly rate-dependent friction. Journal of Geophysical Research: Solid Earth, 101(B11), 25321-25336.

Dal Zilio, L. (2020). Bimodal seismicity in the himalaya controlled by fault friction and geometry. In Cross-scale modeling of mountain building and the seismic cycle: From alps to himalaya (pp. 67-93). Springer.

Day, S. M., Dalguer, L. A., Lapusta, N., \& Liu, Y. (2005). Comparison of finite difference and boundary integral solutions to three-dimensional spontaneous rupture. Journal of Geophysical Research: Solid Earth, 110(B12).

Dieterich, J. H. (1979). Modeling of rock friction: 1. experimental results and constitutive equations. Journal of Geophysical Research: Solid Earth, 84(B5), 21612168. Retrieved from https://agupubs.onlinelibrary.wiley.com/doi/abs/10 .1029/JB084iB05p02161 doi: 10.1029/JB084iB05p02161

Erickson, B. A., \& Dunham, E. M. (2014). An efficient numerical method for earthquake cycles in heterogeneous media: Alternating subbasin and surface-rupturing events on faults crossing a sedimentary basin. Journal of Geophysical Research: Solid Earth, 119(4), 3290-3316. 
Erickson, B. A., Dunham, E. M., \& Khosravifar, A. (2017). A finite difference method for offfault plasticity throughout the earthquake cycle. Journal of the Mechanics and Physics of Solids, 109, 50 - 77. Retrieved from http://www.sciencedirect.com/science/ article/pii/S0022509617305069 doi: https://doi.org/10.1016/j.jmps.2017.08.002

Fialko, Y., Sandwell, D., Agnew, D., Simons, M., Shearer, P., \& Minster, B. (2002). Deformation on nearby faults induced by the 1999 hector mine earthquake. Science, 297(5588), 1858-1862.

Field, E. H., Jordan, T. H., Page, M. T., Milner, K. R., Shaw, B. E., Dawson, T. E., ... others (2017). A synoptic view of the third uniform california earthquake rupture forecast (ucerf3). Seismological Research Letters, 88(5), 1259-1267.

Fumal, T., Weldon, R., Biasi, G., Dawson, T., Seitz, G., Frost, W, \& Schwartz, D. (2002). Evidence for large earthquakes on the san andreas fault at the wrightwood, california, paleoseismic site: Ad 500 to present. Bulletin of the Seismological Society of America, 92(7), 2726-2760.

Grant, L., \& Sieh, K. (1992). Irregular recurrence times and increased seismic hazard from earthquakes on the carrizo segment of the san andreas fault, southern california. In Proc. of the 35th ann. meeting of the assoc. of engineering geologists (pp. 2-9).

Harris, R. A., \& Day, S. M. (1997). Effects of a low-velocity zone on a dynamic rupture. Bulletin of the Seismological Society of America, 87(5), 1267-1280.

Hauksson, E., \& Meier, M.-A. (2019). Applying depth distribution of seismicity to determine thermo-mechanical properties of the seismogenic crust in southern california: comparing lithotectonic blocks. Pure and Applied Geophysics, 176 (3), 1061-1081.

Hillers, G., Ben-Zion, Y., \& Mai, P. M. (2006, January). Seismicity on a fault controlled by rate- and state-dependent friction with spatial variations of the critical slip distance. Journal of Geophysical Research (Solid Earth), 111, B01403. doi: 10.1029/2005JB003859

Huang, Y. (2018). Earthquake rupture in fault zones with along-strike material heterogeneity. Journal of Geophysical Research: Solid Earth.

Huang, Y., \& Ampuero, J.-P. (2011). Pulse-like ruptures induced by low-velocity fault zones. Journal of Geophysical Research: Solid Earth, 116(B12).

Huang, Y., Ampuero, J.-P., \& Helmberger, D. V. (2014). Earthquake ruptures modulated by waves in damaged fault zones. Journal of Geophysical Research: Solid Earth, 119(4), 3133-3154.

Idini, B., \& Ampuero, J.-P. (2020). Fault-zone damage promotes pulse-like rupture and rapid-tremor-reversals.

Jiang, J., \& Lapusta, N. (2016, June). Deeper penetration of large earthquakes on seismically quiescent faults. Science, 352, 1293-1297. doi: 10.1126/science.aaf1496

Kaneko, Y., Ampuero, J.-P., \& Lapusta, N. (2011, October). Spectral-element simulations of long-term fault slip: Effect of low-rigidity layers on earthquake-cycle dynamics. Journal of Geophysical Research (Solid Earth), 116, B10313. doi: 10.1029/2011JB008395

Kaneko, Y., Lapuśta, N., \& Ampuero, J.-P. (2008). Spectral element modeling of spontaneous earthquake rupture on rate and state faults: Effect of velocity-strengthening friction at shallow depths. Journal of Geophysical Research: Solid Earth, 113(B9).

Kim, W., Hong, T.-K., Lee, J., \& Taira, T. (2016). Seismicity and fault geometry of the san andreas fault around parkfield, california and their implications. Tectonophysics, $677,34-44$.

Lapusta, N., \& Rice, J. R. (2003). Nucleation and early seismic propagation of small and large events in a crustal earthquake model. Journal of Geophysical Research: Solid Earth, 108(B4).

Lapusta, N., Rice, J. R., Ben-Zion, Y., \& Zheng, G. (2000). Elastodynamic analysis for slow tectonic loading with spontaneous rupture episodes on faults with rate- and state-dependent friction. Journal of Geophysical Research: Solid Earth, 105(B10), 23765-23789. Retrieved from https://agupubs.onlinelibrary.wiley.com/doi/ abs/10.1029/2000JB900250 doi: 10.1029/2000JB900250

Lewis, M. A., \& Ben-Zion, Y. (2010). Diversity of fault zone damage and trapping structures 
in the parkfield section of the san andreas fault from comprehensive analysis of near fault seismograms. Geophysical Journal International, 183(3), 1579-1595.

Li, Y.-G., \& Leary, P. (1990). Fault zone trapped seismic waves. Bulletin of the Seismological Society of America, 80(5), 1245-1271.

Li, Y.-G., \& Vernon, F. L. (2001). Characterization of the san jacinto fault zone near anza, california, by fault zone trapped waves. Journal of Geophysical Research: Solid Earth, 106(B12), 30671-30688.

Lockner, D. A., Morrow, C., Moore, D., \& Hickman, S. (2011). Low strength of deep san andreas fault gouge from safod core. Nature, $472(7341), 82$.

Ma, X., \& Elbanna, A. (2015). Effect of off-fault low-velocity elastic inclusions on supershear rupture dynamics. Geophysical Journal International, 203(1), 664-677.

Mai, P. M., Spudich, P., \& Boatwright, J. (2005). Hypocenter locations in finite-source rupture models. Bulletin of the Seismological Society of America, 95(3), 965-980.

Marone, C., \& Scholz, C. (1988). The depth of seismic faulting and the upper transition from stable to unstable slip regimes. Geophysical Research Letters, 15(6), 621-624.

Michel, S., Avouac, J.-P., Lapusta, N., \& Jiang, J. (2017). Pulse-like partial ruptures and high-frequency radiation at creeping-locked transition during megathrust earthquakes. Geophysical Research Letters, 44(16), 8345-8351.

Mogi, K. (1962). Magnitude-frequency relation for elastic shocks accompanying fractures of various materials and some related problems in earthquakes. Bull. Earthq. Res. Inst., $40,831-853$.

Parsons, T., Geist, E. L., Console, R., \& Carluccio, R. (2018). Characteristic earthquake magnitude frequency distributions on faults calculated from consensus data in california. Journal of Geophysical Research: Solid Earth, 123(12), 10-761.

Pelties, C., Huang, Y., \& Ampuero, J.-P. (2015). Pulse-like rupture induced by threedimensional fault zone flower structures. Pure and Applied Geophysics, 172(5), 12291241.

Peng, Z., \& Ben-Zion, Y. (2006,/Mar 01). Temporal changes of shallow seismic velocity around the karadere-düzce branch of the north anatolian fault and strong ground motion. pure and applied geophysics, 163(2), 567-600. Retrieved from https:// doi.org/10.1007/s00024-005-0034-6 doi: 10.1007/s00024-005-0034-6

Perfettini, H., Schmittbuhl, J., \& Cochard, A. (2003). Shear and normal load perturbations on a two-dimensional continuous fault: 2. dynamic triggering. Journal of Geophysical Research: Solid Earth, 108(B9).

Perrin, C., Manighetti, I., Ampuero, J.-P., Cappa, F., \& Gaudemer, Y. (2016). Location of largest earthquake slip and fast rupture controlled by along-strike change in fault structural maturity due to fault growth. Journal of Geophysical Research: Solid Earth, 121(5), 3666-3685. Retrieved from https://agupubs.onlinelibrary.wiley.com/ doi/abs/10.1002/2015JB012671 doi: 10.1002/2015JB012671

Powers, P. M., \& Jordan, T. H. (2010). Distribution of seismicity across strike-slip faults in california// Journal of Geophysical Research: Solid Earth, 115(B5).

Rice, J. R. (1993). Spatio-temporal complexity of slip on a fault. Journal of Geophysical Research: Solid Earth, 98(B6), 9885-9907.

Rice, J. R., \& Ben-Zion, Y. (1996). Slip complexity in earthquake fault models. Proceedings of the National Academy of Sciences, 93(9), 3811-3818.

Rivera, L., \& Kanamori, H. (2002). Spatial heterogeneity of tectonic stress and friction in the crust. Geophysical Research Letters, 29(6), 12-1.

Rubin, A., \& Ampuero, J.-P. (2005). Earthquake nucleation on (aging) rate and state faults. Journal of Geophysical Research: Solid Earth, 110(B11).

Ruge, J. W., \& Stüben, K. (1987). Algebraic multigrid. In Multigrid methods (pp. 73-130). SIAM.

Ruina, A. (1983). Slip instability and state variable friction laws. Journal of Geophysical Research: Solid Earth, 88(B12), 10359-10370. Retrieved from https://agupubs .onlinelibrary.wiley.com/doi/abs/10.1029/JB088iB12p10359 doi: 10.1029/ JB088iB12p10359 
Rundle, J. B. (1989). A physical model for earthquakes: 3. thermodynamical approach and its relation to nonclassical theories of nucleation. Journal of Geophysical Research: Solid Earth, 94(B3), 2839-2855. Retrieved from https://agupubs.onlinelibrary .wiley.com/doi/abs/10.1029/JB094iB03p02839 doi: 10.1029/JB094iB03p02839

Rundle, J. B., \& Jackson, D. D. (1977). Numerical simulation of earthquake sequences. Bulletin of the Seismological Society of America, 67(5), 1363-1377.

Rybicki, K., \& Yamashita, T. (2002). On faulting in inhomogeneous media. Geophysical research letters, 29(10).

Scholz, C. (1968). The frequency-magnitude relation of microfracturing in/rock and its relation to earthquakes. Bulletin of the seismological society of America, 58(1), 399415.

Scholz, C. H. (1998). Earthquakes and friction laws. Nature, $391(6662), 37$.

Schwartz, D. P., \& Coppersmith, K. J. (1984). Fault behavior and characteristic earthquakes: Examples from the wasatch and san andreas fault zones. Journal of Geophysical Research: Solid Earth, 89(B7), 5681-5698. Retrieved from https://agupubs .onlinelibrary.wiley.com/doi/abs/10.1029/JB089iB07p05681 doi: 10.1029/ JB089iB07p05681

Seitz, G., Weldon II, R., \& Biasi, G. P. (1997). The pitman canyon paleoseismic record: A re-evaluation of southern san andreas fault segmentation. Journal of Geodynamics, $24(1-4), 129-138$.

Shaw, B. E. (1995). Frictional weakening and slip complexity in earthquake faults. Journal of Geophysical Research: Solid Earth, 100(B9), 18239-18251.

Sibson, R. (1977). Fault rocks and fault mechanisms., Journal of the Geological Society, 133(3), 191-213.

Stanchits, S., Vinciguerra, S., \& Dresen, G. (2006, Jun 01). Ultrasonic velocities, acoustic emission characteristics and crack damage of basalt and granite. pure and applied geophysics, 163(5), 975-994. Retrieved from https://doi.org/10.1007/s00024-006 -0059-5 doi: 10.1007/s00024-006-0059-5

Thurber, C., Roecker, S., Roberts, K., Gold, M., Powell, L., \& Rittger, K. (2003). Earthquake locations and three-dimensional fault zone structure along the creeping section of the san andreas fault near parkfield, ca: Preparing for safod. Geophysical Research Letters, 30(3).

Toké, N. A., Arrowsmith, J. R., Young, J. J., \& Crosby, C. J. (2006). Paleoseismic and postseismic observations of surface slip along the parkfield segment of the san andreas fault. Bulletin of the Seismological Society of America, 96(4B), S221-S238.

Townend, J., \& Zoback, M. D. (2000). How faulting keeps the crust strong. Geology, 28 (5), 399-402.

Unsworth, M. J., Malin, P. E., Egbert, G. D., \& Booker, J. R. (1997). Internal structure of the san andreas fault at parkfield, california. Geology, 25(4), 359-362.

Vidale, J. E., \& Li, Y.-G. (2003). Damage to the shallow landers fault from the nearby hector mine earthquake. Nature, 421(6922), 524-526.

Weng, H., Yang, H., Zhang, Z., \& Chen, X. (2016). Earthquake rupture extents and coseismic slips promoted by damaged fault zones. Journal of Geophysical Research: Solid Earth, 121(6), 4446-4457.

Wesnousky, S. G. (1994). The gutenberg-richter or characteristic earthquake distribution, which is it? Bulletin of the Seismological Society of America, 84 (6), 1940. Retrieved from http://dx.doi.org/

Wessel, P., Smith, W. H., Scharroo, R., Luis, J., \& Wobbe, F. (2013). Generic mapping tools: improved version released. Eos, Transactions American Geophysical Union, $94(45), 409-410$.

Wu, J., Hole, J. A., \& Snoke, J. A. (2010). Fault zone structure at depth from differential dispersion of seismic guided waves: evidence for a deep waveguide on the san andreas fault. Geophysical Journal International, 182(1), 343-354.

Yang, H. (2015). Recent advances in imaging crustal fault zones: A review. Earthquake Science, 28(2), 151-162. 